\title{
Migration strategy and pathogen risk: non-breeding distribution drives malaria prevalence in migratory waders
}

\author{
Nicholas J. Clark, Sonya M. Clegg and Marcel Klaassen \\ N. J. Clark (nicholas.j.clark1214@gmail.com) and Sonya M. Clegg, Environmental Futures Research Inst., School of Environment, Griffith \\ Univ., Gold Coast Campus, QLD 4222, Australia. NJC also at: Natural Environments Program, Queensland Museum, PO Box 3300, South \\ Brisbane, Queensland, 4101, Australia. SMC also at: Edward Grey Inst., Dept of Zoology, Univ. of Oxford, Oxford, OX1 3PS, UK. - M. \\ Klaassen, Centre for Integrative Ecology, School of Life and Environmental Sciences, Deakin Univ., Geelong, VIC, Australia.
}

\begin{abstract}
Pathogen exposure has been suggested as one of the factors shaping the myriad of migration strategies observed in nature. Two hypotheses relate migration strategies to pathogen infection: the 'avoiding the tropics hypothesis' predicts that pathogen prevalence and transmission increase with decreasing non-breeding (wintering) latitude, while the 'habitat selection hypothesis' predicts lower pathogen prevalence in marine than in freshwater habitats. We tested these scarcely investigated hypotheses by screening wintering and resident wading shorebirds (Charadriiformes) for avian malaria blood parasites (Plasmodium and Haemoproteus spp.) along a latitudinal gradient in Australia. We sequenced infections to determine if wintering migrants share malaria parasites with local shorebird residents, and we combined prevalence results with published data in a global comparative analysis. Avian malaria prevalence in Australian waders was 3.56\% and some parasite lineages were shared between wintering migrants and residents, suggesting active transmission at wintering sites. In the global dataset, avian malaria prevalence was highest during winter and increased with decreasing wintering latitude, after controlling for phylogeny. The latitudinal gradient was stronger for waders that use marine and freshwater habitats (marine + freshwater) than for marine-restricted species. Marine + freshwater wader species also showed higher overall avian malaria parasite prevalence than marine-restricted species. By combining datasets in a global comparative analysis, we provide empirical evidence that migratory waders avoiding the tropics during the non-breeding season experience a decreased risk of malaria parasite infection. We also find global support for the hypothesis that marine-restricted shorebirds experience lower parasite pressures than shorebirds that also use freshwater habitats. Our study indicates that pathogen transmission may be an important driver of site selection for non-breeding migrants, a finding that contributes new knowledge to our understanding of how migration strategies evolve.
\end{abstract}

Identifying the factors that cause variation in migration strategies across populations and species is a central goal in ecology and evolution (Gunnarsson et al. 2005, Marra et al. 2015, Shoji et al. 2015). Parasite exposure is hypothesised to be a driver of migration strategies for a diversity of animal hosts, with behaviours or distributions that reduce exposure to parasites thought to convey an adaptive advantage (Altizer et al. 2011, Satterfield et al. 2015). Haemosporidian malaria parasites are vector-borne blood parasites that infect vertebrates across the globe (Schall 2000, Martinsen et al. 2008, Clark et al. 2014, 2015a, Moens and Pérez-Tris 2016). For vector-transmitted parasites, variation in vector encounter rate is a strong determinant of infection risk (Gager et al. 2008, Giles et al. 2014). For instance, saline habitats and low minimum temperatures are considered to be harsh for vector survival and malaria parasite development, leading to reduced malaria prevalence (Figuerola 1999). This spatial variation in malaria prevalence and transmission is predicted to be an important determinant of life-history traits (Lutz et al. 2015), including migratory behaviours (Figuerola and Green 2000).
Avian haemosporidians (Plasmodium and related Haemoproteus spp.; henceforth referred to as 'avian malaria') have received increased attention recently as they are globally distributed and can act as selective agents by reducing host fecundity or causing population declines (Valkiūnas 2005, Lachish et al. 2011, Palinauskas et al. 2011). Moreover, molecular techniques allow for tests of geographical structuring in avian malaria phylogenies that can shed light on areas where transmission occurs for migratory species (Hellgren et al. 2007, Roos et al. 2015). However, the interplay between migration strategies and avian malaria prevalence is understudied, primarily owing to a lack of phylogenetically controlled comparative analyses from widespread host groups (Møller and Szép 2011).

Some of the most impressive avian migrations are exhibited by waders (Charadriiformes), with species and populations travelling up to $22000 \mathrm{~km}$ on trans-equatorial migrations (Gill et al. 2009). Migratory strategies vary dramatically, from species that use both freshwater and saline habitats during the non-breeding season to species that use marine coastlines exclusively (Piersma 1997, 2007). Wader 
migrations also vary within species, with some populations wintering in low-latitude tropical areas while others either extend trans-equatorial migrations to more southern latitudes or remain in temperate northern latitudes to winter ("avoiding the tropics"; Nebel 2007). This variation in nonbreeding habitat and latitude use likely exposes migratory wader populations to different malaria parasite pressures through variation in vector exposure (Piersma 1997, Yohannes et al. 2009).

\section{Hypothetical impacts of wader migration strategies on malaria parasite risk}

Several hypotheses are proposed for how parasite pressure might vary across migration strategies (Piersma 1997, Altizer et al. 2011). Based on the dichotomy of wader distributions, Piersma (1997) formulated the "habitat selection hypothesis" to suggest that species using a broader range of habitats (i.e. using freshwater and marine habitats) are exposed to increased risk of parasite infection. Avian malaria parasites are a tractable model to test this hypothesis, as prevalence can vary across habitats due to climatic variation and vector distributions (Santiago-Alarcon et al. 2012, Loiseau et al. 2013, Sehgal 2015). We therefore expect marine-restricted waders to have lower malaria prevalence compared to species that use freshwater and marine habitats (henceforth marine + freshwater species).

In addition to habitat, the use of different wintering latitudes may influence parasite exposure. It is suggested that migratory waders have reduced parasite infections by using high-latitude breeding sites that remain outside of transmission areas (Piersma 1997). Malaria parasite development is impacted by temperature (Beier 1998), and several studies indicate the prevalence, diversity and transmission of avian malaria are higher in tropical than in temperate regions (Merino et al. 2008, Loiseau et al. 2013, Clark et al. 2014, but see Svensson-Coelho et al. 2014). Because of this low transmission at high latitudes, migratory waders are thought to acquire vector-borne infections predominately at stopovers and non-breeding sites (Piersma 1997, Mendes et al. 2005). If so, we expect that infected wintering migrants will carry similar parasite lineages to local residents, supporting the suggestion that transmission can occur in the non-breeding season. Avoiding the tropics could also benefit non-breeding migrants if the costs of migrating farther past the tropics are offset by low parasite pressure. We therefore expect parasite prevalence to be lower in populations 'avoiding the tropics' by wintering at more southern latitudes.

Molecular studies have examined malaria prevalence for select wader species with different habitat distributions (Yohannes et al. 2009) or within a single flyway (Mendes et al. 2005, D'Amico et al. 2014, Pardal et al. 2014). However, the 'avoiding the tropics hypothesis' remains untested due to a lack of sampling from wintering birds across a latitudinal gradient. In contrast, preliminary support for Piersma's "habitat selection hypothesis" was provided by Mendes et al. (2005), who found significantly higher malaria prevalence in freshwater compared to marine habitats across a narrow latitudinal range in Africa. However, the authors did not distinguish between migrants and year-round residents and, more crucially, the authors deemed their data unsuitable for a phylogenetically controlled analysis due to uneven sampling across habitats (Mendes et al. 2005). Comparative analyses control effects of evolutionary history when testing predictions across species (Pagel 1994), and using such methods can ensure that differences in avian malaria pressure across habitats are not simply a by-product of shared evolutionary histories (Møller and Szép 2011).

Here we test the 'habitat selection' and 'avoiding the tropics' hypotheses using a global comparative analysis of malaria prevalence in waders. We provide data from the previously unsampled East Asian-Australian Flyway by screening resident and wintering waders for parasite infection along a latitudinal gradient in Australia. We use phylogenetic analysis of parasite infections to identify potential transmission areas under the prediction that infected wintering waders will share lineages with local sedentary shorebirds, supporting the suggestion for non-breeding parasite transmission. We then combine our results with published data to generate a global database of wader malaria prevalence. Focussing on migratory species, we use phylogenetic comparative analyses to test two predictions: 1) 'habitat selection': predicts marine-restricted migratory species will exhibit lower prevalence than marine + freshwater species; 2) 'avoiding the tropics': predicts decreased prevalence with increasing wintering latitude.

\section{Methods}

\section{Australian sampling and molecular methods}

Waders were captured using mistnets or cannon nets in Australia in five sampling regions (nine individual sites) across a latitudinal gradient during wintering seasons in 2012 and 2013 (November to March; see Supplementary material Appendix 1 for species and sample site details). Sampling was concentrated around high-density sites ranging from $17^{\circ} \mathrm{S}$ (Broome, Western Australia) to $40^{\circ} \mathrm{S}$ (King Island, Tasmania; Fig. 1, Supplementary material Appendix 1). A blood sample was taken from each bird from the leg or brachial vein and stored as packed red blood cells in ethanol or whole blood in lysis buffer. For birds sampled in southeast Queensland, we also collected thin blood smears to identify whether infections exhibited parasite gametocytes, which would indicate that waders act as 'true' hosts for that particular parasite strain (Valkiūnas et al. 2009, Clark and Clegg 2015). DNA was extracted using ammonium acetate/ethanol precipitation and birds were molecularly sexed following Griffiths et al. (1998) to confirm extraction quality. Samples were screened for malaria DNA (Plasmodium and Haemoproteus spp.) using nested PCR to amplify the parasite cytochrome-b gene (cyt-b), following protocols in Waldenström et al. (2004). We used individual flip-cap PCR tubes to prevent contamination, and we included four positive and two negative controls in each run of $16 \mathrm{sam}$ ples. All samples were screened twice to further minimise false positives and false negatives. None of the negative controls produced amplifications. DNA sequencing was carried out at Macrogen Inc. (Seoul, South Korea) and sequences were compared to the MalAvi database $(<\mathrm{http}: / / \mathrm{mbio}-$ serv2.mbioekol.lu.se/Malavi/ $>$ ) to determine if they were 


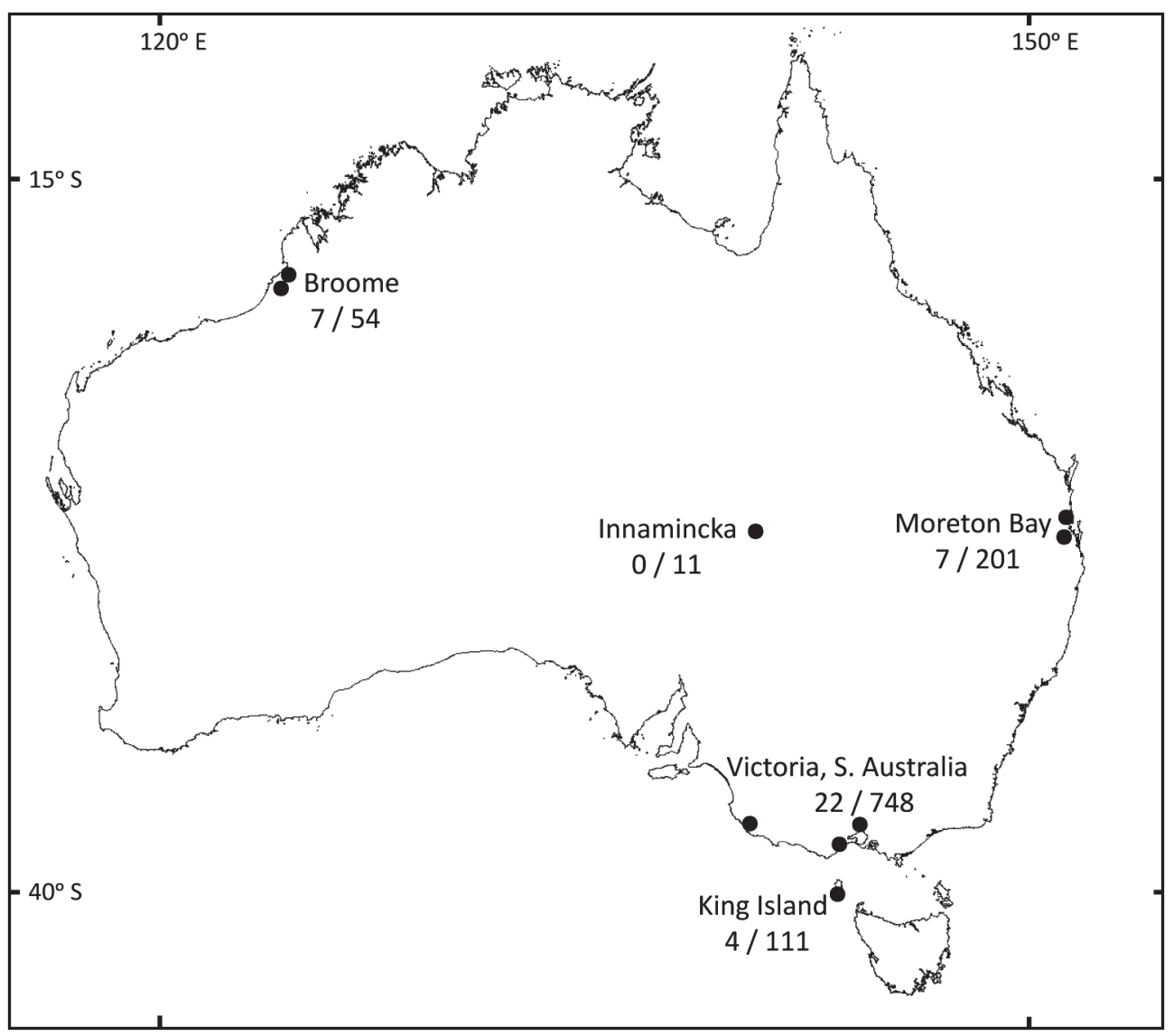

Figure 1. Australian sampling sites (dots) and wader sample sizes (no. of birds infected with avian malaria parasites/total no. of birds sampled). Individual sample sites were grouped into five sampling regions based on proximity and overlap of migratory wader flocks.

new lineages. For samples with mixed infections (carrying both Plasmodium and Haemoproteus), we re-amplified parasite DNA using genus-specific primers (Clark and Clegg unpubl.).

\section{Global dataset of avian malaria prevalence in waders}

We collated a global dataset by combining Australian results with published studies that molecularly screened waders for malaria. We first searched a database of studies that used PCR to screen for malaria in wild birds to November 2012 (Clark et al. 2014). To identify more recent studies, we searched Web of Science, Google Scholar and PubMed following the protocols listed in Clark et al. (2014). Screening of organ tissues can yield important information on the presence of latent parasite infections. However, as the majority of studies only screened blood, we excluded results from organ screening to prevent bias in prevalence estimates (Mendes et al. 2013).

We recorded the following from each site where waders were sampled: absolute latitude, malaria prevalence and total sample size for each species. For each species sampled, we recorded residency as either sedentary (no separation between wintering and breeding grounds) or migratory (spatial separation between wintering and breeding grounds) and in the case of migrants, we recorded stage of annual cycle at time of sampling (breeding, migrating or wintering). In all cases, authors explicitly presented migration status in either the main text or supplementary files (D'Amico and Baker 2010, Pardal et al. 2014). We used species distribution maps to record whether species are restricted to marine habitats (marine) or use both marine and freshwater habitats during the non-breeding season (BirdLife International 2012).

\section{Phylogenetic reconstruction}

We created a molecular wader phylogeny to account for nonindependence of species relatedness. We included species in the molecular alignment if they had at least one sequence from the protein coding mitochondrial genes cytochrome oxidase I and cyt-b (see Supplementary material Appendix 2 for GenBank accession numbers). Sequences were aligned in Genious ver. 5.4 (Drummond et al. 2011) and the gene alignments were concatenated into a single alignment with a total length of $3688 \mathrm{bp}$. We used BEAST ver. 1.6.1 (Drummond and Rambaut 2007) to construct the phylogeny with an uncorrelated lognormal relaxed clock and a Yule prior. We generated two runs of 10000000 generations with sampling at every 10000 generations, and used TRACER ver. 1.6 (<http://tree.bio.ed.ac.uk/software/tracer/ $>)$ to assess convergence. We removed $10 \%$ burn-in from each run and combined runs using LogCombiner ver. 1.4.7 (Drummond and Rambaut 2007). The phylogeny included 39 of the 46 species in the final dataset. We grafted the remaining species onto the most appropriate nodes of the final consensus tree as polytomies using the latest phylogenetic information 
for Charadriiformes (Baker et al. 2007, Mayr 2011). As the resulting tree was not ultrametric, equal branch lengths were set using Grafen's method. The wader phylogeny is presented in Supplementary material Appendix 3.

We constructed molecular phylogenies from parasite cyt-b sequences recovered in Australian samples using identical protocols to the methods outlined above for waders. To investigate whether parasite phylogenies could yield information about where infections were acquired, we included all published avian malaria sequences recovered in Australia and Southeast Asia (see Clark et al. 2015b for details on parasite sequence acquisition). In addition, we included parasite sequences previously recovered from waders and other nonpasserine shorebirds (Mendes et al. 2005, 2013, Levin et al. 2011, 2012) to investigate whether waders in Australia carry similar lineages to shorebirds around the world. Separate phylogenies were constructed for Plasmodium and Haemoproteus spp.

\section{Statistical analyses}

All analyses were carried out in R ver. 3.0.1 (<www.rproject.org $>$ ). We tested for effects of explanatory variables on malaria prevalence in a comparative analysis. We used Bayesian phylogenetically controlled generalised linear mixed models (pGLMMs) in the package 'mcmcGLMM' (Hadfield 2010). The binomial response variable in pGLMMs weights for sample size by including numbers of infected and uninfected individuals for each species. We used weak proper priors with a low degree of belief for all parameters (variance $>0$, degree of belief nu=1). Models were run for 11000000 iterations with a burn-in of 100000 and a thinning value of 1000 . Significance of terms was inferred if the $95 \%$ posterior confidence interval for the coefficient $(\gamma)$ did not overlap with zero and if $P_{M C M C}$-values were equal to or less than 0.05 . We tested all possible combinations of terms and interactions and used deviance information criterion (DIC) to select final models.

Too few individuals from sedentary species were available for inclusion of a 'residency' term in pGLMMs $(n=305)$. Sedentary species were therefore excluded from analyses and are only included in descriptions of overall prevalence and identification of parasite sequences. Using only migrants, the dataset for analyses comprised of 3422 individuals (from 36 species). Additional stressors related to long flights and high densities may result in different disease pressures depending on the stage of the annual cycle (i.e. Breeding versus Migrating versus Wintering; Buehler and Piersma 2008). We therefore fitted a pGLMM with habitat (two levels: marine or marine + freshwater) and migration stage (three levels: breeding, migrating or wintering). We also included mean body mass for each species (from Dunning Jr 2007) as a covariate. We tested all combinations of interactions during model selection. Due to low sample sizes for some species (Supplementary material Appendix 1), we tested the validity of our conclusions by excluding all species with fewer than 15 observations and repeating analyses. This reduced dataset comprised of 3236 individuals from 19 migratory species.

To investigate the 'avoiding the tropics' hypothesis, we tested for a latitudinal gradient in wintering malaria prevalence using a pGLMM that only included wintering migrants $(\mathrm{n}=1730$ individuals; 27 species). Explanatory variables were absolute latitude, habitat, body mass and all interactions. Absolute sampling latitude and body mass were natural $\log$ transformed to reduce the influence of extreme values.

While we obtained sequences from 29 of 40 infections from Australian waders, we did not perform separate analyses for individual parasite genera because most studies published to date have not produced an adequate number of lineages for genus-specific analysis (e.g. Mendes et al. 2005 sequenced seven of 54 infections and recovered a total of three lineages).

\section{Data deposition}

Data available from the Dryad Digital Repository: $<$ http:// dx.doi.org/10.5061/dryad.21s55> (Clark et al. 2015c).

\section{Results}

\section{Avian malaria prevalence in Australian waders}

We sampled 1125 waders in Australia, 54 of which were from low latitudes $\left(17^{\circ}-19^{\circ} \mathrm{S}\right), 212$ from mid latitudes $\left(27^{\circ}-28^{\circ} \mathrm{S}\right)$ and 859 from high latitudes $\left(38^{\circ}-40^{\circ} \mathrm{S}\right.$; Fig. 1, Supplementary material Appendix 1). Samples were taken from 19 species, with 15 species sampled as wintering migrants (Supplementary material Appendix 1). We detected 40 malaria infections for a prevalence of $3.56 \%$ (including migratory and sedentary species). When sites were grouped by latitude category, there was a trend of higher prevalence in low latitudes $(13.0 \%)$ compared to mid- and high-latitudes (3.3\% and $3.0 \%$, respectively), although sample sizes in low latitudes prevented robust comparisons to detect a latitudinal gradient (either for migrants alone or for the full dataset of migratory + sedentary species) due to inadequate mixing of MCMC chains. Prevalence in marine + freshwater migrants was $3.0 \%(n=300$ individuals; five species $)$ and $3.9 \%$ in marine-restricted migrants $(n=665$ individuals; nine species), though the vast majority of marine + freshwater individuals were sampled in mid- and high-latitudes where overall prevalence was low (Supplementary material Appendix 1).

We recovered 13 malaria cyt-b lineages from 29 sequenced infections (Table 1). The remaining 11 infections failed to produce clean sequences due to weak amplifications. Two infections were mixed, containing both Plasmodium and Haemoproteus parasites. The full, un-collapsed parasite phylogenies are presented in Supplementary material Appendix 4 Fig. A1-A2. From 18 sequenced Haemoproteus infections, we recovered four cyt-b lineages (Table 1, Fig. 2). For Plasmodium spp., we recovered nine lineages from 13 infections (Table 1, Fig. A2). False positives were unlikely, as none of the sequences matched sequences from our positive controls and seven of the 13 lineages recovered were new records for GenBank and MalAvi. Stained blood smears were available for seven of the infected birds (representing six lineages), and parasite gametocytes were observed in all seven smears (Table 1).

The four Haemoproteus lineages were all new, and two of these were recovered from both migratory and resident 
Table 1. Avian malaria parasite lineages recovered from waders in Australia. Also presented are the known geographic ranges of lineages based on comparison to the avian malaria database, MalAvi (<http://mbio-serv2.mbioekol.lu.se/Malavi/ $>)$. ${ }^{*}$ indicate lineages that had corresponding blood smears and were confirmed to exhibit gametocytes, suggesting complete parasite development within the host.

\begin{tabular}{|c|c|c|c|c|c|c|}
\hline Lineage & Parasite genus & $\begin{array}{l}\text { GenBank } \\
\text { accession }\end{array}$ & $\begin{array}{c}\text { No. of } \\
\text { infections }\end{array}$ & Migratory wader hosts & $\begin{array}{l}\text { Australian } \\
\text { resident hosts }\end{array}$ & $\begin{array}{c}\text { Countries where malaria } \\
\text { lineage occurs }\end{array}$ \\
\hline BTGOD01* & Plasmodium & KT777732 & 3 & $\begin{array}{l}\text { Limosa lapponica, Tringa } \\
\text { brevipes, Arenaria } \\
\text { interpres }\end{array}$ & none & Australia \\
\hline EMSPO06 & Plasmodium & AB741487 & 1 & Calidris ruficollis & none & $\begin{array}{l}\text { Australia, China, Japan, } \\
\text { South Korea }\end{array}$ \\
\hline FANTAIL01 & Plasmodium & JX021567 & 1 & Calidris tenuirostris & passerines & $\begin{array}{l}\text { Australia, Czech Republic } \\
\text { Singapore }\end{array}$ \\
\hline GRW09 & Plasmodium & FJ404701 & 1 & Charadrius leschenaultii & passerines & $\begin{array}{l}\text { Australia, Cameroon, } \\
\text { Gabon, Malawi, } \\
\text { Netherlands, Nigeria, } \\
\text { Russia, Sweden, } \\
\text { Switzerland, Tanzania, } \\
\text { Ukraine }\end{array}$ \\
\hline GRW15 & Plasmodium & DQ368380.1 & 2 & $\begin{array}{l}\text { Charadrius leschenaultii, } \\
\text { Recurvirostra novaehol- } \\
\text { landiae }\end{array}$ & passerines & Australia, Sweden \\
\hline ORW1* & Plasmodium & AY714203 & 1 & Limosa lapponica & passerines & $\begin{array}{l}\text { Australia, India, Japan, } \\
\text { Myanmar, Papua New } \\
\text { Guinea, Russia, United } \\
\text { Kingdom }\end{array}$ \\
\hline RHIALB02 & Plasmodium & JX418219 & 1 & Arenaria interpres & none & Australia, Philippines \\
\hline STNT01* & Plasmodium & KT777740 & 1 & Calidris ruficollis & none & Australia \\
\hline STNT02* & Plasmodium & KU296187 & 2 & $\begin{array}{l}\text { Calidris ruficollis, Tringa } \\
\text { brevipes }\end{array}$ & none & Australia \\
\hline RUDTURN01 & Haemoproteus & KU296184 & 2 & Arenaria interpres & resident waders & Australia \\
\hline SANDPLOV01* & Haemoproteus & KU296185 & 3 & $\begin{array}{l}\text { Charadrius leschenaultia, } \\
\text { Calidris ruficollis }\end{array}$ & none & Australia \\
\hline SDLG01 & Haemoproteus & KU296186 & 11 & $\begin{array}{l}\text { Calidris alba, Calidris } \\
\text { tenuirostris, Limosa } \\
\text { lapponica, Arenaria } \\
\text { interpres }\end{array}$ & resident waders & Australia \\
\hline TATTLR01* & Haemoproteus & KU296188 & 2 & $\begin{array}{l}\text { Tringa brevipes, Calidris } \\
\text { ruficollis }\end{array}$ & none & Australia \\
\hline
\end{tabular}

waders in Australian wintering sites (RUDTURN01 and SDLG01; Table 1, Fig. 2). Lineage SDLG01 was found in both freshwater and marine-restricted migratory species that co-occur at wintering sites (Table 1). Two sub-genera are currently recognised for Haemoproteus (Haemoproteus and Parahaemoproteus; Valkiūnas 2005), and the four Haemoproteus lineages grouped into a well-supported clade with other non-Passerine waterbird lineages (Pelicaniformes and Charadriiformes hosts) in the Parahaemoproteus clade (Fig. 2, Supplementary material Appendix 1 Fig. A1). Four of the nine Plasmodium lineages recovered in migratory waders have also been found in Australian resident passerines (Table 1). In contrast to Haemoproteus, the Plasmodium lineages did not group together and instead were spread across the phylogeny (Supplementary material Appendix 1 Fig. A2).

\section{Global avian malaria prevalence in migratory waders}

The global dataset, including Australian samples, comprised of 3727 sedentary and migratory waders from 46 species (Supplementary material Appendix 1). Sampling ranged from latitudes $73^{\circ} \mathrm{N}$ to $53^{\circ} \mathrm{S}$ across five continents (North America, South America, Africa, Europe and Australia). The majority of samples were from migrants, with only 305 samples from sedentary waders (Supplementary material Appendix 1). Among migrants, 1730 samples were from wintering birds ( 27 species; sampled from $53^{\circ} \mathrm{N}$ to $40^{\circ}$ ), 1237 from migrating birds ( 13 species; sampled from $53^{\circ} \mathrm{N}$ to $38^{\circ} \mathrm{N}$ ) and 416 from breeding birds (12 species; sampled from $73^{\circ} \mathrm{N}$ to $71^{\circ} \mathrm{N}$ ). Global malaria prevalence (including sedentary and migratory waders) was relatively low and variable among species (mean 6.2 $\pm 1.6 \%$; Supplementary material Appendix 1).

\section{Influence of phylogeny and migration strategies on avian malaria prevalence}

The global dataset provided robust power for comparative analyses (MCMC chains exhibited adequate mixing and autocorrelations for posterior samples were all less than 0.1 . We found a strong influence of phylogeny $(\gamma=8.55$; $95 \%$ $\mathrm{CI}=1.24,21.06)$, an effect that was partially driven by similar habitat and migration strategies for some related species (e.g. both Limosa spp. are migratory marine species, Erythrogonys cinctus and both Vanellus spp. are all resident freshwater species, and four of five species in the Himantopus/Haemo topus/Recurvirostra clade are freshwater users; Supplementary material Appendix 1, 3). However, this phylogenetic effect was also partially due to similarities in malaria prevalence for some related species. Infections were found in 10 of 13 species in the well-sampled Calidris/Arenarial Philomachus clade ( $\mathrm{n}=2815$ individuals), with five of the seven marine + 


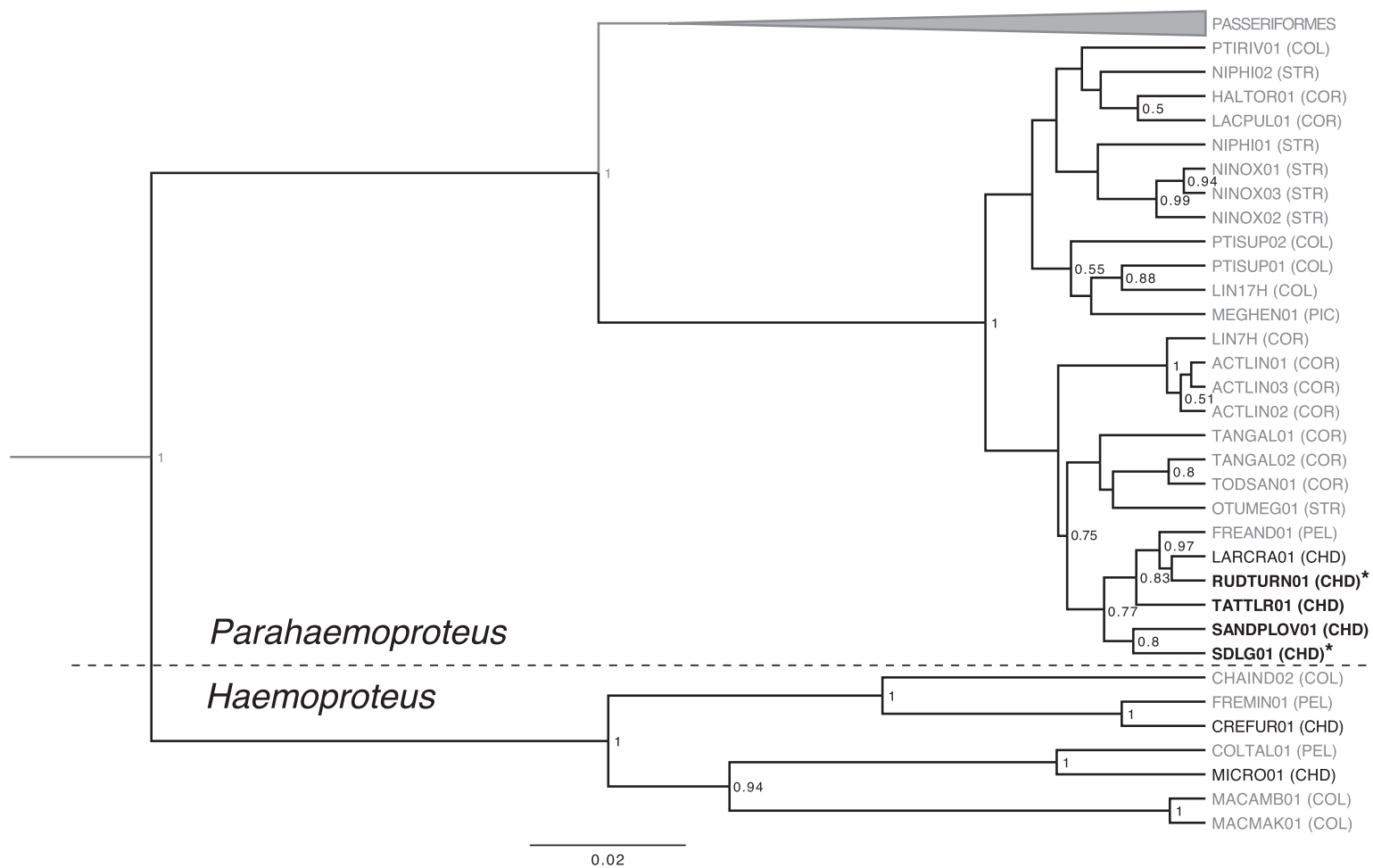

Figure 2. Bayesian molecular phylogeny of Haemoproteus cytochrome-b lineages from the sub-genera Haemoproteus and Parahaemoproteus. All Haemoproteus lineages previously recorded in Australia, China and Southeast Asia were included in the phylogeny. Numbers at nodes indicate Bayesian posterior probabilities. Lineages in black font were recovered from shorebirds in the order Charadriiformes. Lineages in bold were recovered from waders in Australia. Asterisks $\left(^{*}\right)$ indicate lineages shared between resident and migratory waders in Australia. COL, Columbiformes; CHD, Charadriiformes; COR, Coraciiformes; PIC, Piciformes; PEL, Pelicaniformes; STR, Strigiformes.

freshwater species and three of the six marine species exhibiting higher prevalence than the mean for their respective habitat distributions (Supplementary material Appendix 1, 3). In contrast, no infections were found in the three Pluvialis species, though the total sample size for this genus was 18 individuals total.

After accounting for phylogeny, the best model for the migrant dataset included stage of annual cycle and habitat strategy as significant terms. Wintering birds had significantly higher prevalence (mean $8.5 \pm 2.5 \%$ ) than either migrating or breeding birds (mean $0.7 \pm 0.6 \%$ versus $0.3 \pm 0.2 \%$, respectively; $\gamma=4.19 ; 95 \% \mathrm{CI}=0.53,8.14 ; \mathrm{p}_{M C M C}=0.01$; Fig. 3a). We found global comparative support for Piersma's "habitat selection hypothesis", with marine-restricted species exhibiting significantly lower malaria prevalence than marine+freshwater species (mean $2.3 \pm 0.9 \%$ versus $5.9 \pm 1.7 \%$, respectively; $\gamma=-2.04 ; 95 \% \mathrm{CI}=-4.03$, $-0.03 ; \mathrm{p}_{M C M C}=0.04 ;$ Fig. $3 \mathrm{~b}$ ). When excluding species with
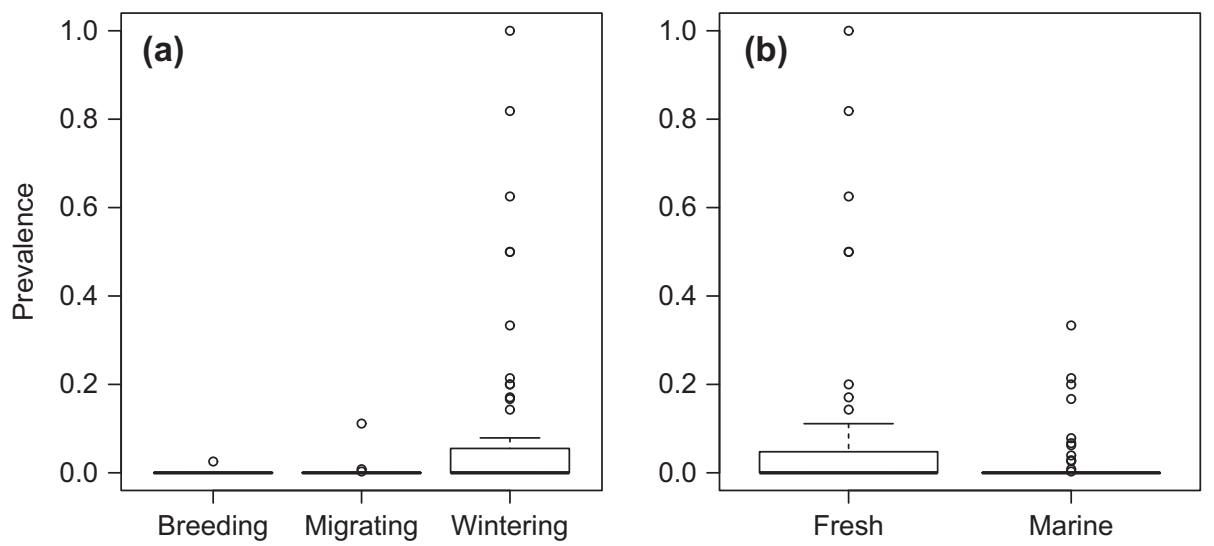

Figure 3. Avian malaria parasite prevalence in migratory waders (a) at different migratory stages and (b) with different habitat selection strategies. Box and whisker plots indicate third quartiles (hinges of box) and $95 \%$ confidence intervals (error bars) of the median prevalence. Fresh $=$ wader species that use freshwater and marine habitats during migration and winter; Marine $=$ wader species that are restricted to marine habitats during migration and winter. 
fewer than 15 samples, MCMC chains mixed adequately and effect sizes for migration stage and habitat were smaller but were still significant (see Supplementary material Appendix 5 for model statistics).

\section{Avoiding the tropics: a latitudinal gradient in avian malaria prevalence}

When considering only wintering migrants, phylogeny again had a strong influence $(\gamma=11.05 ; 95 \% \mathrm{CI}=1.47,27.35)$ and the best model included significant terms for habitat and sampling latitude (Supplementary material Appendix 5). Malaria prevalence decreased significantly with increasing wintering latitude $(\gamma=-6.48 ; 95 \% \mathrm{CI}=-11.22,-1.77$; $\left.\mathrm{p}_{M C M C}=0.001\right)$ and was significantly lower in marine than marine + freshwater species $(\gamma=-18.05 ; 95 \% \mathrm{CI}=-37.00$, $\left.-0.01 ; \mathrm{p}_{M C M C}=0.04\right)$. An interaction between latitude and habitat reduced model deviance and was borderline significant $\left(\gamma=5.53 ; 95 \% \mathrm{CI}=-0.19,11.94 ; \mathrm{p}_{M C M C}=0.053\right)$. Model predictions indicate the latitudinal gradient was stronger for marine + freshwater species (Fig. 4). Body mass and all other interactions did not significantly influence prevalence or improve model deviance. Results were similar when excluding species with low sample size, although $\mathrm{p}_{M C M C}$ values for habitat and the habitat $\times$ latitude interaction were marginally non-significant $\left(\mathrm{p}_{M C M C}=0.06\right.$ for each effect, Supplementary material Appendix 5).

\section{Discussion}

Using a global dataset that includes the previously unsampled Australian flyway, we present the first evidence that waders 'avoiding the tropics' during winter experience a decreased risk of malaria parasite infection. The latitudinal gradient was stronger for marine + freshwater species, suggesting that wintering farther from the tropics may not benefit all populations equally. We also provide comparative evidence that species using both marine and freshwater habitats have higher parasite prevalence than marine-restricted species, supporting Piersma's 'habitat selection hypothesis'. Migratory populations with different non-breeding distributions may be exposed to different interspecific interactions, a finding that has implications for our understanding of how migration strategies evolve.

\section{High malaria prevalence in winter: implications for avoiding the tropics}

Our results show that waders experience higher malaria burdens in winter compared to migration and breeding periods. We also found that migrants are carrying a diversity of parasite lineages, some of which are shared with residents in the wintering range. Indeed, six of the 13 recovered parasite lineages have also been found in Australian resident birds, indicating that these lineages can be actively transmitted on the wintering grounds. Although only seven infected waders had corresponding blood smears, all seven showed parasite gametocytes and were therefore confirmed as true infections that were transmissible to vectors. Our results support the suggestion that long-distance migrants may be susceptible to infections following arrival to wintering grounds, perhaps because their immune function can be compromised from migration-induced fatigue or muscle injury (Piersma 1997, Klaassen et al. 2000). Moreover, the recovery of a well-supported Haemoproteus clade that is only currently known to infect shorebirds, including two lineages that were shared between migratory and resident waders, suggests that migrants play a role in shaping the population dynamics of these parasites. In contrast to Haemoproteus spp., Plasmodium lineages in Australasia are typically host-generalists with wide geographic ranges (Clark et al. 2014, 2015b, Clark and Clegg 2015), making it difficult to determine where transmission occurred. For instance, Plasmodium lineages ORW1 and EMSPO06 have both been found infecting resident birds throughout Australasia (Ishtiaq et al. 2007,



Figure 4. Avian malaria parasite prevalence across wintering latitudes (natural log transformed) for migratory waders with differing habitat strategies: (a) species that utilise freshwater and marine habitats; (b) species that are restricted to marine habitats. Regression lines are phylogenetically-controlled MCMCglmm predictions with $95 \%$ confidence intervals (shaded areas). To improve interpretation, data points are scaled according to sample size. . 
Huang et al. 2015), and so these parasites may have been picked up either during a migratory stopover or after arrival in Australia. The presence of latent infections could also be problematic for discovering transmission areas, as parasites can be missing from the blood and will not be amplified during PCR. However, our conclusion that winter represents an increased risk of infection does not hinge on distinguishing between recently acquired and re-activated infections. Latent infections cannot be picked up by a vector, and so the fact that active infections are more abundant in winter is good evidence that wintering birds face higher parasite pressures due to an increased chance of infections being passed around the population (Begon et al. 2002).

We also find evidence that increased wintering latitude results in decreased malaria prevalence, supporting our prediction that populations that 'avoid the tropics' experience decreased risk of infection. A similar trend was observed for tundra swans in Alaska, with populations that spend more time near the tropics exhibiting increased malaria prevalence (Ramey et al. 2012). We propose that shorebird populations wintering farther from the tropics are exposed to fewer disease-carrying vectors, as both vector diversity and the rate of within-vector parasite development are reduced in high latitudes (Rueda 2008, Loiseau et al. 2013). Because pathogen infection can reduce body condition and delay arrival times (Møller et al. 2004, Bradley and Altizer 2005), risk of infection may place additional pressures on migrants to choose the best non-breeding sites. Collectively, our findings support a broader recognition that choosing a non-breeding site is an important and complex behavioural trait that might have impacts throughout the life cycle (Gunnarsson et al. 2005, Marra et al. 2015, Shoji et al. 2015).

\section{Support for Piersma's habitat selection hypothesis}

Our results also support the hypothesis that marine-restricted waders are using habitats with relatively low malaria parasite pressures (Piersma 1997, Mendes et al. 2005, Yohannes et al. 2009). Moreover, our study is the first to highlight a difference in parasite prevalence in wintering birds with different habitat choices, as marine species showed a weaker latitudinal gradient in wintering malaria prevalence than marine + freshwater species. Although the vectors that transmit avian malaria are poorly studied (Lima and Bensch 2014), evidence increasingly suggests that most vectors rely on permanent or semi-permanent freshwater to breed (Kalluri et al. 2007, Njabo et al. 2009, Santiago-Alarcon et al. 2012, Valkiūnas et al. 2015). The higher incidence of malaria infections in marine + freshwater wader species implicates the availability of vector breeding sites as a strong determinant of parasite risk. For freshwater habitats, changes in latitude may correlate with changes in climate that can influence the prevalence and diversity of parasites (Sehgal et al. 2010, Oakgrove et al. 2014). Climate influences may be less apparent for marine habitats, where lack of freshwater and high salt spray will be similarly unfavourable to vectors at different latitudes (Figuerola 1999). Consequently, marine-restricted species may minimize overall parasite exposure by migrating between Arctic breeding areas and coastal habitats (Alerstam et al. 2003), and could experience fewer disease-related benefits from avoiding the tropics than marine + freshwater species. Nevertheless, our comparison of latitudinal trends for marine and freshwater species was not statistically significant and so should be interpreted with appropriate caution.

\section{Influence of phylogeny on malaria parasite prevalence in waders}

In their study of the 'habitat selection hypothesis', Mendes et al. (2005) were unable to use a comparative analysis, a limitation that was recognised by the authors and was highlighted by Møller and Szép (2011). While we encountered similar problems analysing the Australian subset, our global analysis suggests that evolutionary history can influence malaria prevalence for some species. Infections were commonly found in species belonging to the Calidris/ArenarialPhilomachus clade, with the majority of species showing above average prevalence for their respective habitats. While it is unclear if certain shared traits predispose species to malaria, it is possible that this high prevalence is due to microhabitat use or foraging characteristics that expose species to high vector densities. For instance, the marine-restricted Arenaria interpres regularly scavenges around rotting organic material and may experience unusually high pathogen exposure (Mendes et al. 2006), while Philomachus pugnax often uses rice fields in Africa that remain wet and could adequately maintain vectors during the dry winter season (Mendes et al. 2013). Alternatively, closely related species could have similar immune strategies that allow them to better tolerate malaria infections, though this idea warrants future study.

\section{Importance of sample size and the biological significance of malaria in waders}

Although comparative pGLMMs weight for samples size and our results were robust when excluding poorly sampled species, we stress the importance of large sample sizes for parasite screening in wildlife. Despite extensive sampling, migratory marine + freshwater species were represented by only 300 samples from five species in Australia, the vast majority of which were sampled at high latitudes where prevalence is likely to be lower. Monitoring for natural prevalence levels is crucial in disease ecology (Grogan et al. 2014, Clark et al. 2015b), and sample size is an important consideration for identifying populations at risk.

The influence of animal dispersal on disease incidence has gained increased recognition of late (Altizer et al. 2011, Møller and Szép 2011, Jenkins et al. 2012, Ramey et al. 2012, Bauer et al. 2015, Clark and Clegg 2015, Cohen et al. 2015). Some migration strategies are thought to be the result of species actively avoiding parasite-rich habitats (Piersma 1997, Qviller et al. 2013, Sandström et al. 2013), suggesting that migrants must balance the costs of extra migration distances with the potential benefits of reduced pathogen exposure. Despite the low overall prevalence in our dataset, we found that nearly $10 \%$ of waders wintering in low latitude sites were infected with malaria across the globe. We propose that malaria in waders may be more common than previously supposed (Greiner et al. 1975), indicating that these parasites could have important but overlooked biological significance. However, the low overall parasite prevalence in 
our study raises questions about how important malaria parasites are for shaping migratory patterns in waders. A prevalence of $10 \%$ in tropical sites may not be adequate to act as a strong selective pressure and might not outweigh the added benefits of wintering in the tropics, such as the reduced flight distances (Klaassen 1996, Somveille et al. 2015). Moreover, we have no conclusive evidence for pathological effects of malaria parasites in shorebirds (but see Mendes et al. 2013), and it seems likely that any impacts might differ between Plasmodium and Haemoproteus parasites (Valkiūnas 2005). A multiplicity of variables can shape migration patterns in birds (Klaassen et al. 2000, 2012, Bauer et al. 2010, Duriez et al. 2012), and the relative importance of malaria parasites compared to these factors as drivers of migratory routes requires further consideration.

Acknowledgements - We thank the Australian Wader Study Group and numerous volunteers for invaluable support in sample collection. Queensland fieldwork was completed under Queensland DERM permit WISP10823212 and Griffith University Ethics Approval ENV/01/12/AEC. Laboratory work and Moreton Bay fieldwork was supported by grants to N. Clark from Birds Queensland and BirdLife Australia. SMC was funded by an ERC Advanced Grant to Tim Coulson, MK by funds from the US National Institutes of Health (HHSN266200700010C) and the Australian Research Council (DP130101935).

\section{References}

Alerstam, T. et al. 2003. Long-distance migration: evolution and determinants. - Oikos 103: 247-260.

Altizer, S. et al. 2011. Animal migration and infectious disease risk. - Science 331: 296-302.

Baker, A. J. et al. 2007. Phylogenetic relationships and divergence times of Charadriiformes genera: multigene evidence for the Cretaceous origin of at least 14 clades of shorebirds. - Biol. Lett. 3: 205-210.

Bauer, S. et al. 2010. Many routes lead to Rome: potential causes for the multi-route migration system of red knots, Calidris canutus islandica. - Ecology 91: 1822-1831.

Bauer, S. et al. 2015. Timing is crucial for consequences of migratory connectivity. - Oikos DOI: 10.1111/oik.02706.

Begon, M. et al. 2002. A clarification of transmission terms in host-microparasite models: numbers, densities and areas. - Epidemiol. Infection 129: 147-153.

Beier, J. C. 1998. Malaria parasite development in mosquitoes. - Annu. Rev. Entomol. 43: 519-543.

BirdLife International 2012. Bird species distribution maps of the world. Ver. 2.0.

Bradley, C. A. and Altizer, S. 2005. Parasites hinder monarch butterfly flight: implications for disease spread in migratory hosts. - Ecol. Lett. 8: 290-300.

Buehler, D. M. and Piersma, T. 2008. Travelling on a budget: predictions and ecological evidence for bottlenecks in the annual cycle of long-distance migrants. - Phil. Trans. R. Soc. B 363: 247-266.

Clark, N. J. and Clegg, S. M. 2015. The influence of vagrant hosts and weather patterns on the colonization and persistence of blood parasites in an island bird. - J. Biogeogr. 42: 641-651.

Clark, N. J. et al. 2014. A review of global diversity in avian haemosporidians (Plasmodium and Haemoproteus: Haemosporida): new insights from molecular data. - Int. J. Parasitol. 44: 329-338.
Clark, N. J. et al. 2015a. Molecular and morphological characterization of Haemoproteus (Parahaemoproteus) ptilotis, a parasite infecting Australian honeyeaters (Meliphagidae), with remarks on prevalence and potential cryptic speciation. - Parasitol. Res. 114: 1921-1928.

Clark, N. J. et al. 2015b. Specialist enemies, generalist weapons and the potential spread of exotic pathogens: malaria parasites in a highly invasive bird. - Int. J. Parasitol. 45: 891-899.

Clark, N. J. et al. 2015c. Data from: Migration strategy and pathogen risk: non-breeding distribution drives malaria prevalence in migratory waders. - Dryad Digital Repository, < http:// dx.doi.org/10.5061/dryad.21s55>.

Cohen, E. B. et al. 2015. Avian migrants facilitate invasions of Neotropical ticks and tick-borne pathogens into the United States. - Appl. Environ. Microbiol. in press.

D'Amico, V. L. and Baker, A. J. 2010. A rare case of Plasmodium (Haemamoeba) relictum infection in a free-living red knot (Calidris canutus rufa, Scolopacidae). - J. Ornithol. 151: 951-954.

D'Amico, V. L. et al. 2014. Multi-year surveillance of selected avian pathogens in the migrant shorebird red knot (Calidris canutus rufa) at its main stopover site in Patagonia, Argentina. - J. Ornithol. 155: 555-559.

Drummond, A. J. and Rambaut, A. 2007. BEAST: Bayesian evolutionary analysis by sampling trees. - BMC Evol. Biol. 7: 214. DOI: $10.1186 / 1471-2148-1187-1214$.

Drummond, A. J. et al. 2011. Geneious ver. 5.4.

Dunning Jr, J. B. 2007. CRC handbook of avian body masses, 2nd edn. - CRC Press.

Duriez, O. et al. 2012. Comparing the seasonal survival of resident and migratory oystercatchers: carry-over effects of habitat quality and weather conditions. - Oikos 121: 862-873.

Figuerola, J. 1999. Effects of salinity on rates of infestation of waterbirds by haematozoa. - Ecography 22: 681-685.

Figuerola, J. and Green, A. J. 2000. Haematozoan parasites and migratory behaviour in waterfowl. - Evol. Ecol. 14: $143-153$

Gager, A. B. et al. 2008. Do mosquitoes filter the access of Plasmodium cytochrome b lineages to an avian host? - Mol. Ecol. 17: 2552-2561.

Giles, J. R. et al. 2014. Invasive potential of cattle fever ticks in the southern United States. - Paras. Vect. 7: 189.

Gill, R. E. et al. 2009. Extreme endurance flights by landbirds crossing the Pacific Ocean: ecological corridor rather than barrier? - Proc. R. Soc. B 276: 447-457.

Greiner, E. C. et al. 1975. Distribution of the avian hematozoa of North America. - Can. J. Zool. 53: 1762-1787.

Griffiths, R. et al. 1998. A DNA test to sex most birds. - Mol. Ecol. 7: 1071-1075.

Grogan, L. F. et al. 2014. Surveillance for emerging biodiversity diseases of wildlife. - PLoS Pathol. 10: e1004015.

Gunnarsson, T. G. et al. 2005. Seasonal matching of habitat quality and fitness in a migratory bird. - Proc. R. Soc. B. 272: 2319-2323.

Hadfield, J. D. 2010. MCMC methods for multi-response generalized linear mixed models: the MCMCglmm R package. - J. Stat. Softw. 33: 1-22.

Hellgren, O. et al. 2007. Detecting shifts of transmission areas in avian blood parasites - a phylogenetic approach. - Mol. Ecol. 16: $1281-1290$.

Huang, X. et al. 2015. Genetic diversity, temporal dynamics, and host specificity in blood parasites of passerines in north China. - Parasitol. Res. 114: 4513-4520.

Ishtiaq, F. et al. 2007. Prevalence and diversity of avian hematozoan parasites in Asia: a regional survey. - J. Wildl. Dis. 43: 382-398. 
Jenkins, T. et al. 2012. Migratory behaviour of birds affects their coevolutionary relationship with blood parasites. - Evolution 66: 740-751.

Kalluri, S. et al. 2007. Surveillance of arthropod vector-borne infectious diseases using remote sensing techniques: a review. - PLoS Pathol. 3: e116.

Klaassen, M. 1996. Metabolic constraints on long-distance migration in birds. - J. Exp. Biol. 199: 57-64.

Klaassen, M. et al. 2000. Flight costs and fuel composition of a bird migrating in a wind tunnel. - Condor 102: 444-451.

Klaassen, M. et al. 2012. Ecophysiology of avian migration in the face of current global hazards. - Phil. Trans. R. Soc. B 367: 1719-1732.

Lachish, S. et al. 2011. Fitness effects of endemic malaria infections in a wild bird population: the importance of ecological structure. - J. Anim. Ecol. 80: 1196-1206.

Levin, I. I. et al. 2011. Hippoboscid-transmitted Haemoproteus parasites (Haemosporida) infect Galapagos Pelecaniform birds: evidence from molecular and morphological studies, with a description of Haemoproteus iwa. - Int. J. Parasitol. 41: 1019-1027.

Levin, I. I. et al. 2012. Novel Haemoproteus species (Haemosporida: Haemoproteidae) from the swallow-tail gull (Lariidae), with remarks on host range of Hippoboscid-transmitted avian Haemoproteids. - J. Parasitol. 98: 847-854.

Lima, M. and Bensch, S. 2014. Why some parasites are widespread and abundant while others are local and rare? - Mol. Ecol. 23: 3130-3132.

Loiseau, C. et al. 2013. Predictions of avian Plasmodium expansion under climate change. - Sci. Rep. 3: 1126.

Lutz, H. L. et al. 2015. Parasite prevalence corresponds to host life history in a diverse assemblage of Afrotropical birds and haemosporidian parasites. - PLoS ONE 10: e0121254.

Marra, P. P. et al. 2015. Non-breeding season habitat quality mediates the strength of density-dependence for a migratory bird. - Proc. R. Soc. B 282 doi: 10.1098/rspb.2015.0624.

Martinsen, E. S. et al. 2008. A three-genome phylogeny of malaria parasites (Plasmodium and closely related genera): evolution of life-history traits and host switches. - Mol. Phylogenet. Evol. 47: 261-273.

Mayr, G. 2011. The phylogeny of charadriiform birds (shorebirds and allies)-reassessing the conflict between morphology and molecules. - Zool. J. Linn. Soc. 161: 916-934.

Mendes, L. et al. 2005. Disease-limited distributions? Contrasts in the prevalence of avian malaria in shorebird species using marine and freshwater habitats. - Oikos 109: 396-404.

Mendes, L. et al. 2006. Variation in the innate and acquired arms of the immune system among five shorebird species. - J. Exp. Biol. 209: 284-291.

Mendes, L. et al. 2013. Hidden haemosporidian infections in ruffs (Philomachus pugnax) staging in northwest Europe en route from Africa to Arctic Europe. - Parasitol. Res. 112: 2037-2043.

Merino, S. et al. 2008. Haematozoa in forest birds from southern Chile: latitudinal gradients in prevalence and parasite lineage richness. - Aust. Ecol. 33: 329-340.

Moens, M. A. and Pérez-Tris, J. 2016. Discovering potential sources of emerging pathogens: South America is a reservoir of generalist avian blood parasites. - Int. J. Parasitol. 46: 41-49.

Møller, A. and Szép, T. 2011. The role of parasites in ecology and evolution of migration and migratory connectivity. - J. Ornithol. 152: 141-150.

Møller, A. P. et al. 2004. Parasitism, immunity, and arrival date in a migratory bird, the barn swallow. - Ecol. 85: 206-219.

Nebel, S. 2007. Differential migration of shorebirds in the East Asian-Australasian Flyway. - Emu 107: 14-18.
Njabo, K. Y. et al. 2009. Coquillettidia (Culicidae, Diptera) mosquitoes are natural vectors of avian malaria in Africa. - Malaria J. 8.

Oakgrove, K. S. et al. 2014. Distribution, diversity and drivers of blood-borne parasite co-infections in Alaskan bird populations. - Int. J. Parasitol. 44: 717-727.

Pagel, M. 1994. Detecting correlated evolution on phylogenies: a general method for the comparative analysis of discrete characters. - Proc. R. Soc. B 255: 37-45.

Palinauskas, V. et al. 2011. Plasmodium relictum (lineage SGS1) and Plasmodium ashfordi (lineage GRW2): the effects of the co-infection on experimentally infected passerine birds. - Exp. Parasitol. 127: 527-533.

Pardal, S. et al. 2014. Shorebird low spillover risk of mosquitoborne pathogens on Iberian wetlands. - J. Ornithol. 155: 549-554.

Piersma, T. 1997. Do global patterns of habitat use and migration strategies co-evolve with relative investments in immunocompetence due to spatial variation in parasite pressure? - Oikos 80: 623-631.

Piersma, T. 2007. Using the power of comparison to explain habitat use and migration strategies of shorebirds worldwide. - J. Ornithol. 148: 45-59.

Qviller, L. et al. 2013. Landscape level variation in tick abundance relative to seasonal migration in red deer. - PloS ONE 8: e71299.

Ramey, A. M. et al. 2012. Molecular detection of hematozoa infections in tundra swans relative to migration patterns and ecological conditions at breeding grounds. - PloS ONE 7: e45789.

Roos, F. et al. 2015. Prevalence and diversity of avian malaria parasites in migratory black skimmers (Rynchops niger, Laridae, Charadriiformes) from the Brazilian Amazon Basin. - Parasitol. Res. 114: 3903-3911.

Rueda, L. M. 2008. Global diversity of mosquitoes (Insecta: Diptera: Culicidae) in freshwater. - Hydrobiologia 595: 477-487.

Sandström, C. A. et al. 2013. Latitudinal variability in the seroprevalence of antibodies against Toxoplasma gondii in non-migrant and Arctic migratory geese. - Vet. Parasitol. 194: 9-15.

Santiago-Alarcon, D. et al. 2012. Diptera vectors of avian Haemosporidian parasites: untangling parasite life cycles and their taxonomy. - Biol. Rev. 87: 928-964.

Satterfield, D. A. et al. 2015. Loss of migratory behaviour increases infection risk for a butterfly host. - Proc. R. Soc. B 282 doi: 10.1098/rspb.2014.1734.

Schall, J. 2000. Transmission success of the malaria parasite Plasmodium mexicanum into its vector: role of gametocyte density and sex ratio. - Parasitology 121: 575-580.

Sehgal, R. N. 2015. Manifold habitat effects on the prevalence and diversity of avian blood parasites. - Int. J. Parasitol: Parasitol. Wild. 4: 421-430.

Sehgal, R. N. M. et al. 2010. Spatially explicit predictions of blood parasites in a widely distributed African rainforest bird. - Proc. R. Soc. B 278: 1025-1033.

Shoji, A. et al. 2015. Breeding phenology and winter activity predict subsequent breeding success in a trans-global migratory seabird. - Biol. Lett. 11: 20150671.

Somveille, M. et al. 2015. Why do birds migrate? A macroecological perspective. - Glob. Ecol. Biog. 24: 664-674.

Svensson-Coelho, M. et al. 2014. Reciprocal specialization in multihost malaria parasite communities of birds: a temperatetropical comparison. - Am. Nat. 184: 624-635.

Valkiūnas, G. 2005. Avian malaria parasites and other Haemosporida. - CRC Press.

Valkiūnas, G. et al. 2009. Nested cytochrome $b$ polymerase chain reaction diagnostics detect sporozoites of hemosporidian 
parasites in peripheral blood of naturally infected birds. - J. Parasitol. 95: 1512-1515.

Valkiūnas, G. et al. 2015. Complete sporogony of Plasmodium relictum (lineage pGRW4) in mosquitoes Culex pipiens pipiens, with implications on avian malaria epidemiology. - Parasitol. Res. 114: 3075-3085.

Supplementary material (available online as Appendix oik03220 at <www.oikosjournal.org/appendix/oik-03220>). Appendix 1. Sampling locations and haemosporidian prevalence for waders in the final global dataset. Appendix 2 . GenBank accession numbers for sequences used in the wader phylogeny. Appendix 3. Molecular wader phylogeny used for phylogenetic MCMCglmms. Appendix 4. Full molecular parasite phylogenies for Haemoproteus and Plasmodium parasite lineages. Appendix 5. Model statistics for initial and final pGLMMs.
Waldenström, J. et al. 2004. A new nested polymerase chain reaction method very efficient in detecting Plasmodium and Haemoproteus infections from avian blood. - J. Parasitol. 90: 191-194.

Yohannes, E. et al. 2009. Prevalence of malaria and related haemosporidian parasites in two shorebird species with different winter habitat distribution. - J. Ornithol. 150: 287-291. 